TITLE:

\title{
Magnetization modeling of silicon steel using a simplified domain structure model
}

\author{
$\operatorname{AUTHOR}(\mathrm{S}):$
}

Sudo, M.; Matsuo, T.

\section{CITATION:}

Sudo, M.... [et al]. Magnetization modeling of silicon steel using a simplified domain structure model. JOURNAL OF APPLIED PHYSICS 2012, 111(7): 07D107.

ISSUE DATE:

2012-04

URL:

http://hdl.handle.net/2433/160648

\section{RIGHT:}

Copyright 2012 American Institute of Physics. This article may be downloaded for personal use only. Any other use requires prior permission of the author and the American Institute of Physics. The following article appeared in JOURNAL OF APPLIED PHYSICS 111, 07 D107 (2012) and may be found at http://link.aip.org/link/?jap/111/07D107 


\section{AIP $\mid$ Appolied Physics}

\section{Magnetization modeling of silicon steel using a simplified domain structure model}

M. Sudo and T. Matsuo

Citation: J. Appl. Phys. 111, $07 D 107$ (2012); doi: 10.1063/1.3672073

View online: http://dx.doi.org/10.1063/1.3672073

View Table of Contents: http://jap.aip.org/resource/1/JAPIAU/v111/i7

Published by the American Institute of Physics.

\section{Related Articles}

Current-induced synchronized switching of magnetization

Appl. Phys. Lett. 101, 062408 (2012)

Modeling and simulation of high voltage and radio-frequency transformer

J. Appl. Phys. 111, 07E737 (2012)

Enhancement of perpendicular magnetic anisotropy in FeB free layers using a thin MgO cap layer

J. Appl. Phys. 111, 07C723 (2012)

Effect of enhanced damping caused by spin-motive force on vortex dynamics

J. Appl. Phys. 111, 07D120 (2012)

Extended frequency analysis of magnetic losses under rotating induction in soft magnetic composites

J. Appl. Phys. 111, 07E325 (2012)

\section{Additional information on J. Appl. Phys.}

Journal Homepage: http://jap.aip.org/

Journal Information: http://jap.aip.org/about/about_the_journal

Top downloads: http://jap.aip.org/features/most_downloaded

Information for Authors: http://jap.aip.org/authors

\section{ADVERTISEMENT}

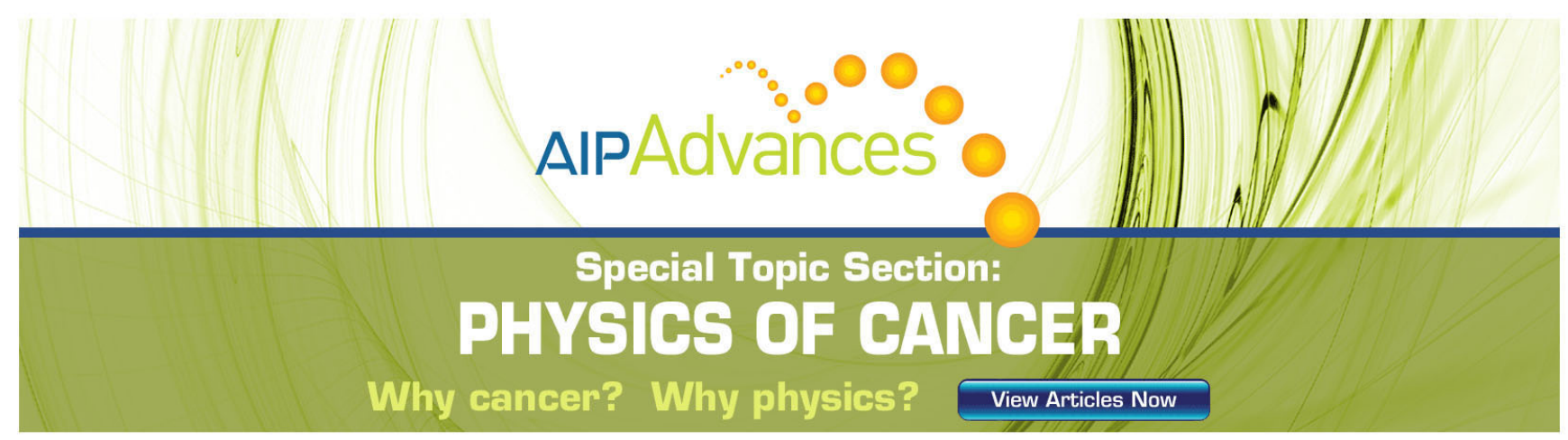




\title{
Magnetization modeling of silicon steel using a simplified domain structure model
}

\author{
M. Sudo and T. Matsuo a) \\ Graduate School of Engineering, Kyoto University, Kyotodaigaku-katsura, Nishikyo-ku, Kyoto, 615-8510, \\ Japan
}

(Presented 3 November 2011; received 23 September 2011; accepted 23 October 2011; published online 22 February 2012)

\begin{abstract}
A simplified domain structure model with shape anisotropy and cubic crystalline anisotropy is applied to the analysis of the mesoscopic magnetization of silicon steel sheets. Alternating magnetization is analytically described by the transition among several magnetization states, such as the single-domain, $180^{\circ}$ domain-wall-motion, and magnetization-rotation types. The magnetic field vector is calculated from a given average magnetization vector under the rotational magnetization condition. The loci of the magnetic field vector qualitatively reconstruct the rotational magnetization properties of non-oriented and grain-oriented silicon steel sheets. (C) 2012 American Institute of Physics. [doi:10.1063/1.3672073]
\end{abstract}

\section{INTRODUCTION}

To represent vector hysteretic magnetization, many vector hysteresis models consist of an assembly of vector hysteresis units called hysterons. ${ }^{1,2}$ The hysteron is often assumed to be a single-magnetic-domain particle. However, mesoscopic or macroscopic magnetization is usually governed by multi-domain structures. As a result, many hysterons are required to simulate domain-wall motion.

As a mesoscopic magnetic model, the domain-structure model (DSM) ${ }^{3-5}$ has been proposed and successfully applied to the analysis of magnetic thin films. A simplified version of the $\mathrm{DSM}^{6}$ reveals the basic mechanisms of mesoscopic magnetization including domain-wall motion and magnetization rotation. Accordingly, the assembly of the simplified DSM (SDSM) is expected to constitute an efficient physical macroscopic magnetization model.

This paper applies the SDSM to the analysis of the magnetization of silicon steel sheets. To examine the possibility of the SDSM being a building block of a macroscopic magnetization model, the magnetization of a grain-oriented steel sheet is analyzed, which is difficult to do with vector hysteresis models because of strong anisotropy.

\section{SIMPLIFIED DOMAIN STRUCTURE MODEL}

An SDSM$^{6}$ with two domains is examined in analyzing a grain-oriented silicon steel sheet. The rolling and transverse directions (RD and TD) are along the $x$ - and $y$-directions, respectively. Each domain $i(i=1,2)$ has uniform magnetization; the normalized magnetization vector is given by $\boldsymbol{m}_{i}=\left(\sin \theta_{i} \cos \phi_{i}, \sin \theta_{i} \sin \phi_{i}, \cos \theta_{i}\right)$.

The total magnetic energy to be minimized is normalized by the crystalline anisotropic energy. The normalized total energy, $e$, is given as,

$$
e=e_{\mathrm{ap}}+e_{\mathrm{an}}+e_{\mathrm{w}}+e_{\mathrm{st}}
$$

\footnotetext{
${ }^{\text {a)} E l e c t r o n i c ~ m a i l: ~ t m a t s u o @ k u e e . k y o t o-u . a c . j p . ~}$
}

where $e_{\mathrm{ap}}$ is the Zeeman energy, $e_{\mathrm{an}}$ is the anisotropic energy, $e_{\mathrm{w}}$ is the domain-wall energy, and $e_{\mathrm{st}}$ is the magnetostatic energy.

Similar to the case for the grain-oriented silicon steel sheet, the three axes of easy magnetization are set along the $(1,0,0),(0,1,1)$, and $(0,-1,1)$ directions. Thus, the normalized cubic crystalline anisotropic energy is given as,

$$
\begin{aligned}
e_{\mathrm{an}}= & \sum_{i=1}^{2} \lambda_{i}\left[\sin ^{2} \theta_{i} \cos ^{2} \phi_{i}\left(1-\cos ^{2} \phi_{i} \sin ^{2} \theta_{i}\right)\right. \\
& \left.+\frac{1}{4}\left(\cos ^{2} \theta_{i}-\sin ^{2} \theta_{i} \sin ^{2} \phi_{i}\right)^{2}\right]
\end{aligned}
$$

where $\lambda_{1}$ and $\lambda_{2}=1-\lambda_{1}$ are the volume ratios of domains 1 and 2 , respectively.

The Zeeman energy due to the normalized applied field, $\boldsymbol{h}=h\left(\cos \phi_{H}, \sin \phi_{H}, 0\right)$ is given as,

$$
e_{\text {ap }}=-2 \boldsymbol{h} \cdot\left(\lambda_{1} \boldsymbol{m}_{1}+\lambda_{2} \boldsymbol{m}_{2}\right),
$$

where $h=H_{\mathrm{ap}} / \kappa M_{\mathrm{s}}, H_{\mathrm{ap}}$ is the magnitude of the applied magnetic field, $M_{\mathrm{s}}$ is the magnitude of spontaneous magnetization, $\kappa=2 K_{1} /\left(\mu_{0} M_{\mathrm{s}}^{2}\right)$, and $K_{1}$ is the anisotropy constant.

A simple Bloch wall model gives the domain-wall energy as,

$$
e_{\mathrm{w}}=\frac{w}{2}\left(1-\boldsymbol{m}_{\mathbf{1}} \cdot \boldsymbol{m}_{\mathbf{2}}\right),
$$

where $w=4 l_{k} / D, l_{k}=\sqrt{A / K_{1}}$, and $D$ is the width of the two domains. The parameter, $w$, represents the energy cost to have a domain wall.

The SDSM assumes that the demagnetization field is uniformly given by the multiplication of demagnetizing factors and the average magnetization. The magnetostatic energy is given as,

$$
e_{\mathrm{st}}=s_{x} m_{x}^{2}+s_{y} m_{y}^{2}+s_{z} m_{z}^{2},
$$




$$
\left(m_{x}, m_{y}, m_{z}\right)=\lambda_{1} \boldsymbol{m}_{1}+\lambda_{2} \boldsymbol{m}_{2},
$$

where $s_{x}=k_{x} / \kappa, s_{y}=k_{y} / \kappa$, and $s_{z}=k_{z} / \kappa: k_{x}, k_{y}$, and $k_{z}$ are the demagnetizing factors. Because of the sheet structure, $s_{z}$ is assumed very large compared with $s_{x}$ and $s_{y}$.

The magnetization is determined by finding $\boldsymbol{X}=\left(\theta_{1}, \phi_{1}, \theta_{2}, \phi_{2}, \lambda_{1}\right)$ that locally minimizes the total energy, $e$. The total energy becomes a local extremum when

$$
\partial e / \partial \boldsymbol{X}=0 .
$$

The solution to Eq. (7) gives a local minimum when all eigenvalues of the Hessian matrix, $\partial^{2} e / \partial \boldsymbol{X}^{2}$, are positive.

\section{MAGNETIZATION ANALYSIS FOR AN ALTERNATING MAGNETIC FIELD}

\section{A. Magnetization along the rolling direction}

The magnetization process along the RD is examined with $\phi_{H}=0$. Figure 1 shows the magnetization curves along the $\mathrm{RD}$ with $w=0.01$ and $\left(s_{x}, s_{y}, s_{z}\right)=(5,5,100)$, $(0.5,5,100)$, where $m_{x}$, given by Eq. (6), is plotted. The dominant stable solutions are classified into two types:

$\mathrm{S}$ is the single-domain type, where $\theta_{1}=\theta_{2}=\pi / 2$, $\phi_{1}=\phi_{2}$, and $\lambda_{1}=1 / 2$; and

WM is the $180^{\circ}$ domain-wall motion type, where $\theta_{1}=\theta_{2}=\pi / 2$ and $\left(\phi_{1}, \phi_{2}\right)=(0, \pi)$ or $(\pi, 0)$.

Stable solutions in Fig. 1 have the labels "S" and "WM," corresponding to the preceding classification.

When $\phi_{H}=0$, Eq. (7) has an S-type solution of $\boldsymbol{X}=(\pi / 2,0, \pi / 2,0,1 / 2)$, that yields $\partial e^{2} / \partial^{2} \boldsymbol{X}$ eigenvalues of $1+h-s_{x}+s_{y}, 1+h-s_{x}+s_{z}$, and $1+h-s_{x}+w$. Consequently, the $\mathrm{S}$-type solution is stable when,

$$
h>h_{\mathrm{s}}=\max \left(s_{x}-s_{y}-1, s_{x}-s_{z}-1, s_{x}-w-1\right),
$$

which agrees with the S-type solutions in Fig. 1. When $\phi_{H}=0$, Eq. (7) has a WM-type solution, $\boldsymbol{X}=(\pi / 2,0$, $\left.\pi / 2, \pi, h / 2 s_{x}+1 / 2\right)$, that yields the linear magnetization,

$$
m_{x}=h / s_{x} .
$$

The WM-type solution is stable when

$$
|h|<h_{\mathrm{WM}} \equiv \min \left(s_{x} \sqrt{\frac{1+s_{y}-w}{1+s_{y}+s_{y} w}}, \quad s_{x} \sqrt{\frac{1+s_{z}-w}{1+s_{z}+s_{z} w}}\right) .
$$

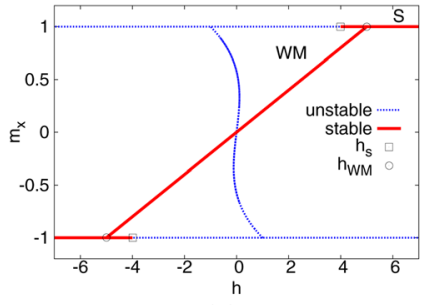

(a)

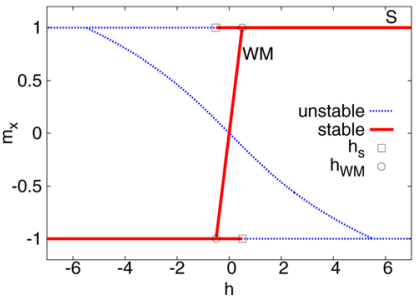

(b)
FIG. 1. (Color online) Magnetization curves along the RD with $w=0.01$ and $s_{z}=100$ (solid lines: stable; dashed lines: unstable): (a) $\left(s_{x}, s_{y}\right)=(5,5)$, and (b) $\left(s_{x}, s_{y}\right)=(0.5,5)$.

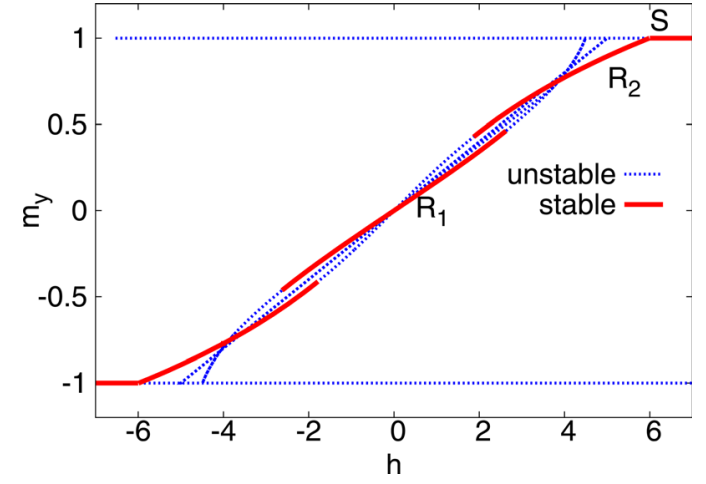

FIG. 2. (Color online) Magnetization curve along the TD with $w=0.01$ and $\left(s_{x}, s_{y}, s_{z}\right)=(0.5,5,100)$ (solid lines: stable; dashed lines: unstable).

\section{B. Magnetization along the transverse direction}

The magnetization process along the TD is examined with $\phi_{H}=\pi / 2$. Figure 2 shows the magnetization curves along the TD with $w=0.01$ and $\left(s_{x}, s_{y}, s_{z}\right)=(0.5,5,100)$ where $m_{y}$, given by Eq. (7), is plotted. When $\phi_{H}=\pi / 2$, two types of magnetization rotation are dominantly observed:

$\mathrm{R}_{1}: \theta_{1}=\theta_{2}=\pi / 2, \phi_{2}=\pi-\phi_{1}$, and $\lambda_{1}=1 / 2$ when $h$ is small; and

$\mathrm{R}_{2}: \theta_{2}=\pi-\theta_{1}, \phi_{1}=\phi_{2}= \pm \pi / 2$, and $\lambda_{1}=1 / 2$ after the $\mathrm{R}_{1}$ solution becomes unstable owing to the large value of $h$.

Stable solutions in Fig. 2 have the labels " $\mathrm{R}_{1}$ " and " $\mathrm{R}_{2}$," corresponding to the preceding classification.

The $\mathrm{R}_{1}$-type solution satisfies

$$
h=\left(-\frac{3}{2} m_{y}^{2}+1+s_{y}-w\right) m_{y} .
$$

The $\mathrm{R}_{2}$-type solution satisfies

$$
h=\left(2 m_{y}^{2}-1+s_{y}-w\right) m_{y} .
$$

\section{ANALYSIS OF ROTATIONAL MAGNETIZATION}

The silicon steel sheet is often excited with sinusoidal magnetic flux density but not with a sinusoidal magnetic field, which largely means that the average magnetization

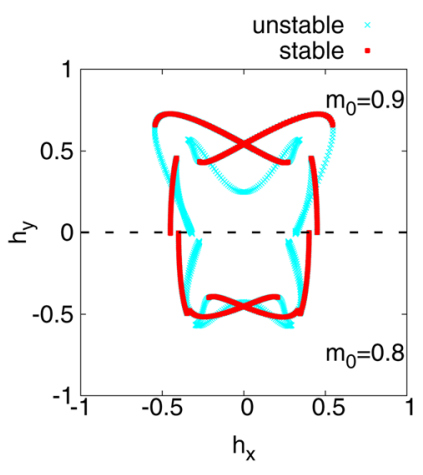

(a)

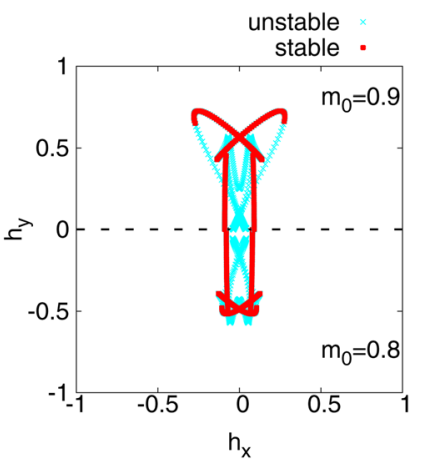

(b)
FIG. 3. (Color online) Loci of $\left(h_{x}, h_{y}\right)$ under the rotational magnetization conditions with $m_{0}=0.8$ (lower), 0.9 (upper): (a) $\left(s_{x}, s_{y}\right)=(0.5,0.5)$, and (b) $\left(s_{x}, s_{y}\right)=(0.1,0.5)$. 


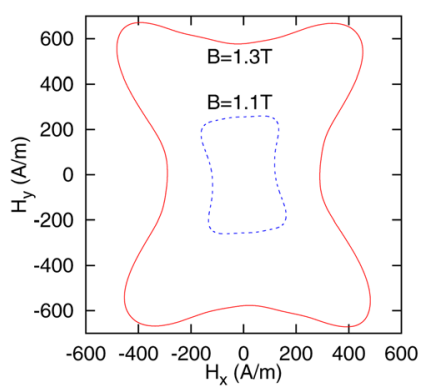

(a)

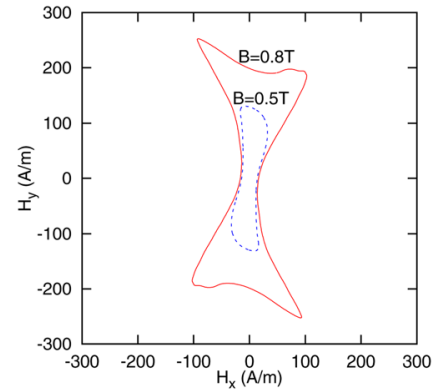

(b)
FIG. 4. (Color online) Loci of the measured magnetic field vector of silicon steel sheets under a condition of rotational magnetic flux: (a) non-oriented silicon steel sheet, and (b) grain-oriented silicon steel sheet.

sinusoidally changes and that $h$ and $\phi_{H}$ are unknowns for the DSM. When the normalized rotational magnetization is given by $m_{0}\left(\cos \phi_{M}, \sin \phi_{M}, 0\right)$, the equations to be solved are,

$$
\begin{gathered}
\boldsymbol{f}\left(\boldsymbol{X}, h, \phi_{H}\right) \\
=\left(\begin{array}{c}
\partial e / \partial \boldsymbol{X} \\
\lambda_{1} \sin \theta_{1} \cos \phi_{1}+\lambda_{2} \sin \theta_{2} \cos \phi_{2}-m_{0} \cos \phi_{M} \\
\lambda_{1} \sin \theta_{1} \sin \phi_{1}+\lambda_{2} \sin \theta_{2} \sin \phi_{2}-m_{0} \sin \phi_{M} \\
\lambda_{1} \cos \theta_{1}+\lambda_{2} \cos \theta_{2},
\end{array}\right)=0,
\end{gathered}
$$

where $m_{0}$ and $\phi_{M}$ are given parameters. Figure 3 portrays the loci of $\left(h_{x}, h_{y}\right)=\left(h \cos \phi_{H}, h \sin \phi_{H}\right)$ under the rotational magnetization condition when $\left(w, s_{z}\right)=(0.01,100)$, $m_{0}=0.8,0.9$, and

(a) there is no shape anisotropy: $\left(s_{x}, s_{y}\right)=(0.5,0.5)$; and

(b) there is shape anisotropy: $\left(s_{x}, s_{y}\right)=(0.1,0.5)$.

The effect of cubic crystalline anisotropy becomes large for large $m_{0}$. The cubic anisotropy results in a strong applied magnetic field, $h$, along the directions of $\pm 55^{\circ}$ and $\pm 125^{\circ}$. The shape anisotropy requires high $h$ along the $y$-direction. Figure 4 portrays the loci of the measured magnetic field vectors of non-oriented and grain-oriented silicon steel sheets under a condition of rotational magnetic flux. The loci in Fig. 3(a) are similar to those in Fig. 4(a), which suggests that the angular dependence in the crystal grain distribution is not weak in the non-oriented silicon steel sheet. The loci in Fig. 3(b) are similar to those in Fig. 4(b), which means that the strong anisotropy of the grain-oriented steel sheet is due to not only the crystalline anisotropy, but also the shape anisotropy resulting from the anisotropic crystal grain shape. This qualitative agreement suggests that the SDSM can be used as a building block of a more macroscopic magnetization model. However, the anisotropy of the grain-oriented steel sheet may be due to the magnetoelastic energy, which will be reported upon in the near future.

\section{CONCLUSION}

The SDSM qualitatively represents the alternating magnetization of a grain-oriented silicon steel sheet. The analytically obtained magnetization curves show that the magnetization along the $\mathrm{RD}$ is dominated by $180^{\circ}$ domainwall motion and single-domain types, whereas two types of magnetization rotation are dominant along the TD.

The magnetic field vector computationally obtained under a rotational magnetization condition qualitatively reconstructs the rotational magnetization properties of nonoriented and grain-oriented silicon steel sheets. This suggests that the SDSM can be used as a building block of a more macroscopic magnetization model.

Future work remains to introduce the magnetoelastic energy and domain-wall pinning for a more realistic representation of the magnetization.

\section{ACKNOWLEDGMENTS}

This work was supported in part by the JFE 21st Century Foundation through Technical Research Aid and by the Japan Society for the Promotion of Science through a Grant-in-Aid for Scientific Research (C) (Grant No. 23560328).

${ }^{1}$ E. C. Stoner and E. P. Wohlfarth, Philos. Trans. R. Soc. London, Ser. A 240, 599 (1948).

${ }^{2}$ E. Cardelli, E. D. Torre, and A. Faba, J. Appl. Phys. 103, 07 D927 (2008).

${ }^{3}$ N. Smith, IEEE Trans. Magn. 24, 2380 (1988).

${ }^{4}$ C. Saka, K. Shiiki, and K. Shinagawa, J. Appl. Phys. 68, 263 (1990).

${ }^{5}$ T. Matsuo, N. Mimuro, and M. Shimasaki, J. Magn. Magn. Mater. 320, e1029 (2008)

${ }^{6}$ T. Matsuo, J. Appl. Phys. 109, 07D332 (2011). 